QUARTERLY OF APPLIED MATHEMATICS

VOLUME LXX, NUMBER 3

SEPTEMBER 2012, PAGES 597-611

S 0033-569X(2012)01314-3

Article electronically published on May 9, 2012

\title{
CAN CONSTITUTIVE RELATIONS BE REPRESENTED BY NON-LOCAL EQUATIONS?
}

\author{
BY \\ TOMMASO RUGGERI \\ Department of Mathematics $\&$ Research Center of Applied Mathematics, University of Bologna, Via \\ Saragozza 8, 40123 Bologna, Italy
}

Dedicated to Constantine Dafermos for his 70th birthday

\begin{abstract}
Using the modern theory of extended thermodynamics, it is possible to show that the well-known constitutive equations of continuum mechanics in non-local form with respect to space variables such as Fourier's, Navier-Stokes's, Fick's and Darcy's laws are in reality an approximation of the general balance laws when some suitable relaxation times are neglected. In the present paper we conjecture that this fact is completely general and indeed all the "real" constitutive equations of mathematical physics are local in nature and, therefore, the corresponding differential systems of balance equations are hyperbolic rather than parabolic. This does not means that non-local equations are not useful not only because there are situations where non-local equations may be an effective approximation, but also because non-locality permits us to obtain the evaluation of non-observable quantities such as the velocity and the temperature of each constituent of a mixture of fluids. An important consequence is that these equations do not need to satisfy the so-called objectivity principle that on the contrary still continues to be valid only for the constitutive equations. We prove that under suitable assumptions the conditions dictated by the entropy principle in the hyperbolic case guarantee the validity of the entropy principle also in the parabolic limit. Considerations are also made with regard to the formal limit between hyperbolic systems and parabolic ones and from hyperbolic versus hyperbolic, between a system and a subsystem. We end the paper with a discussion of the main analytical properties concerning the global existence of smooth solutions for dissipative hyperbolic systems.
\end{abstract}

1. Balance laws and constitutive equations. The physical laws in continuum theories are balance laws: Let $\mathbf{F}^{0}(\mathbf{x}, t), \mathbf{x} \in \Omega, t \in R^{+}$be a generic $R^{N}$-vector of densities.

Received January 19, 2012.

2010 Mathematics Subject Classification. Primary 54C40, 14E20; Secondary 46E25, 20C20.

Key words and phrases. Constitutive equations, objective principle, hyperbolic systems of balance laws. The author was supported in part by GNFM of INdAM.

E-mail address: tommaso.ruggeri@unibo.it

(C)2012 Brown University 
Then we have:

$$
\frac{d}{d t} \int_{\Omega} \mathbf{F}^{0} d \Omega=-\int_{\Sigma} \mathbf{G}^{i} n_{i} d \Sigma+\int_{\Omega} \mathbf{f} d \Omega
$$

where the first integral on the r.h.s. represents the fluxes of some quantities $\mathbf{G}^{i} \in$ $R^{N},(i=1,2,3)$ through the surface $\Sigma$ of unit normal $\mathbf{n} \equiv\left(n_{i}\right)$ and velocity $\mathbf{v} \equiv\left(v_{i}\right)$, while the last integral represents the productions.

Under regularity assumptions the system (1.1) can be put in the local form:

$$
\frac{\partial \mathbf{F}^{0}}{\partial t}+\frac{\partial \mathbf{F}^{i}}{\partial x^{i}}=\mathbf{f}, \quad \mathbf{F}^{i}=\mathbf{F}^{0} v^{i}+\mathbf{G}^{i}
$$

For example in the case of continuum mechanics the balance laws are:

$$
\begin{array}{ll}
\frac{\partial \rho}{\partial t}+\frac{\partial \rho v_{i}}{\partial x^{i}}=0 & \text { (mass conservation) }, \\
\frac{\partial\left(\rho v_{j}\right)}{\partial t}+\frac{\partial}{\partial x^{i}}\left(\rho v_{i} v_{j}-t_{i j}\right)=\rho b_{j} & \text { (momentum balance) } \\
\frac{\partial E}{\partial t}+\frac{\partial}{\partial x^{i}}\left(E v_{i}+q_{i}-t_{i j} v_{j}\right)=\rho b_{i} v_{i}+r & \text { (energy balance) },
\end{array}
$$

where $E=\rho v^{2} / 2+\rho \varepsilon$ and $\rho, \mathbf{t} \equiv\left(t_{i j}\right), \mathbf{q} \equiv\left(q_{i}\right), \varepsilon$ are respectively the mass density, the stress tensor, the heat flux and the internal energy. The stress tensor can be decomposed as $\mathbf{t}=-p \mathbf{I}+\boldsymbol{\sigma}$, where $p$ is the pressure and $\boldsymbol{\sigma} \equiv\left(\sigma_{i j}\right)$ is the viscous stress tensor. Moreover $\mathbf{b} \equiv\left(b_{i}\right)$ and $r$ are respectively the body force and the heat supply. When $\mathbf{b}$ and $r$ vanish the balance laws become a system of conservation laws.

In order to have a closed system we need the so-called constitutive equations. A very rough mathematical definition of constitutive equations can be considered as the equations that need to close the system; i.e., choosing an independent field $\mathbf{u} \in R^{N}$ we have to give the relations between the $5 N$ components of the vectors $\mathbf{F}^{0}, \mathbf{F}^{i}$ and $\mathbf{f}$ and the $N$ field components of the unknown vector $\mathbf{u}$. But of course, as we will see later, this definition has no physical meaning because we need that the additive equations must represent the real constitutive properties of the material.

For a long time the constitutive equations were made in an empirical way and they belong substantially to the following three big classes:

- Local constitutive equations. Examples are:

- stress-strain relations in non-linear elasticity $\mathbf{t} \equiv \mathbf{t}(\mathbf{E})$ (Hooke's law in the linear case);

- The caloric and thermal equations of state in Euler fluids that connect internal energy and pressure as functions of density and temperature

$$
\varepsilon \equiv \varepsilon(\rho, T), \quad p \equiv p(\rho, T) .
$$

Introducing these constitutive equations in the balance laws we obtain a differential system, which, in general, is hyperbolic.

- Non-local type (in space). In the case of a single dissipative fluid, examples are:

- the Fourier's law $\mathbf{q}=L \operatorname{grad}(1 / T)$, where $L$ is related with the heat conductivity;

- the Navier-Stokes equation $\boldsymbol{\sigma}=2 \nu \mathbf{D}^{D}+\lambda \operatorname{div} \mathbf{v} \mathbf{I}$, where $\nu$ and $\lambda$ are phenomenological viscosity coefficients, while $\mathbf{D}=(1 / 2)\left(\nabla \mathbf{v}+(\nabla \mathbf{v})^{T}\right)$ is the 
deformation velocity, and $\mathbf{D}^{D}$ indicates the deviatoric part of $\mathbf{D}$ that is traceless.

- In the case of a mixture of dissipative fluids with $n$ constituents, well-known examples are the Navier-Stokes's, Fourier's and Fick's laws:

$$
\begin{aligned}
& \boldsymbol{\sigma}=2 \nu \mathbf{D}^{D}+\lambda \operatorname{div} \mathbf{I}, \\
& \mathbf{q}=L \operatorname{grad}\left(\frac{1}{T}\right)+\sum_{b=1}^{n-1} L_{b} \operatorname{grad}\left(\frac{\mu_{b}-\mu_{n}}{T}\right), \\
& \mathbf{J}_{a}=\tilde{L}_{a} \operatorname{grad}\left(\frac{1}{T}\right)-\sum_{b=1}^{n-1} L_{a b} \operatorname{grad}\left(\frac{\mu_{b}-\mu_{n}}{T}\right),
\end{aligned}
$$

where the $\mu$ 's are the chemical potentials and

$$
\tilde{L}_{a}, L_{b}, L_{a b} \quad(a, b=1,2, \ldots, n-1)
$$

are phenomenological coefficients related to diffusion.

When we introduce these constitutive equations in the balance laws we obtain a system of differential equations where the spatial derivatives are of second order and the time derivatives are of first order. These differential systems have a parabolic structure.

- Non-local type (in time). Examples are: the visco-elasticity or, in general, all materials in which the stress depends not only on deformation but also on the history of the deformation (constitutive equations with memory). Except for the case of an exponential kernel the mathematical structure of the system is of integro-differential type.

2. Universal principle for constitutive equations. In the modern constitutive theory all the constitutive equations must obey two universal principles:

- The objectivity principle: the balance laws are invariant with respect to Galilean transformations, and the proper constitutive equations are independent of the Observer (material frame indifference, Noll; see, e.g., [1]);

- The entropy principle: in Rational Thermodynamics any solutions of the full system satisfy a supplementary entropy balance law with a non-negative entropy production (Coleman-Noll [2]; Müller [3]):

$$
\frac{\partial \rho S}{\partial t}+\frac{\partial}{\partial x^{i}}\left(\rho S v^{i}+\Phi^{i}\right) \geq 0 \text { for all processes, }
$$

where $S$ denotes the entropy density and $\Phi^{i}$ the entropy flux.

3. The frame-dependence of the heat flux. A long debate existed in the literature after Ingo Müller published a famous paper [4] in which he proved that the Fourier and Navier-Stokes "constitutive" equations violate the objectivity principle. At that time Müller was convinced that the result indicates that the objectivity principle is not a valid principle and therefore a huge literature started between supporter and not supporter of the objectivity principle. Several authors added also artificial time derivatives to try to recover the objectivity for the heat equation and for the stress. Here we record an 
independent observation on the subject by Bressan [5] and Ruggeri [6] that may solve in a simple way the problem. Bressan and Ruggeri observed that a possible interpretation of Müller's result is that the objectivity principle is indeed universal, but the Fourier and Navier-Stokes equations are not "true" constitutive equations. The precise and convincing answer was done by the Extended Thermodynamics (ET). To understand this fundamental point let us recall briefly the main ingredients of ET [7].

4. The extended thermodynamics. The kinetic theory describes the state of a rarefied gas through the phase density $f(\mathbf{x}, t, \mathbf{c})$, where $f(\mathbf{x}, t, \mathbf{c}) d \mathbf{c}$ is the number density of atoms at point $\mathbf{x}$ and time $t$ that have velocities between $\mathbf{c}$ and $\mathbf{c}+d \mathbf{c}$. The phase density obeys the Boltzmann equation

$$
\frac{\partial f}{\partial t}+c^{i} \frac{\partial f}{\partial x^{i}}=Q
$$

where $Q$ represents the collisional terms. The macroscopic thermodynamic quantities are identified as moments of the phase density

$$
F_{k_{1} k_{2} \cdots k_{j}}=\int f c_{k_{1}} c_{k_{2}} \cdots c_{k_{j}} d c
$$

and due to the Boltzmann equation (4.1), the moments satisfy an infinity hierarchy of balance laws with increasing tensorial index in which the flux in one equation becomes the density in the next one:

$$
\begin{aligned}
& \partial_{t} F+\partial_{i} F_{i}=0 \\
& \swarrow \\
& \partial_{t} F_{k_{1}}+\partial_{i} F_{i k_{1}}=0 \\
& \swarrow \\
& \partial_{t} F_{k_{1} k_{2}}+\partial_{i} F_{i k_{1} k_{2}}=P_{\left\langle k_{1} k_{2}\right\rangle} \\
& \swarrow \\
& \partial_{t} F_{k_{1} k_{2} k_{3}}+\partial_{i} F_{i k_{1} k_{2} k_{3}}=P_{k_{1} k_{2} k_{3}} \\
& \vdots \\
& \partial_{t} F_{k_{1} k_{2} \ldots k_{n}}+\partial_{i} F_{i k_{1} k_{2} \ldots k_{n}}=P_{k_{1} k_{2} \ldots k_{n}}
\end{aligned}
$$

By taking into account that $P_{k k}=0$, the first five equations are conservation laws and coincide with the mass, momentum and energy conservations (1.3), while the remaining ones are balance laws.

When we consider a theory where only the first $n$ moments are considered of physical relevance and we cut the hierarchy at the density with tensor of rank $n$, we encounter the problem of closure because the last flux and the production terms are not in the list of the densities.

The idea of Rational Extended Thermodynamics [7] was to view the truncated system on the same ground of a phenomenological system of continuum mechanics and then 
consider the new quantities as constitutive functions:

$$
\begin{aligned}
& F_{k_{1} k_{2} \ldots k_{n} k_{n+1}} \equiv F_{k_{1} k_{2} \ldots k_{n} k_{n+1}}\left(F, F_{k_{1}}, F_{k_{1} k_{2}}, \ldots F_{k_{1} k_{2} \ldots k_{n}}\right) \\
& P_{k_{1} k_{2} \ldots k_{j}} \equiv P_{k_{1} k_{2} \ldots k_{j}}\left(F, F_{k_{1}}, F_{k_{1} k_{2}}, \ldots F_{k_{1} k_{2} \ldots k_{n}}\right) ; \quad 2 \leq j \leq n .
\end{aligned}
$$

In accordance with the rational continuum theory, the restrictions must be imposed only on the basis of universal principles, i.e.: Entropy principle, Objectivity Principle and Causality and Stability (convexity of the entropy).

The restrictions are so strong (in particular the entropy principle) that, at least, for processes not too far from equilibrium, the system is completely closed. In the case of 13 moments the results are in perfect agreement with the kinetic closure procedure proposed by Grad [8] (see [9], 7]).

To avoid cumbersome algebra we restrict our attention to the Grad equations only in the one-dimensional case

$$
\left\{\begin{array}{l}
\dot{\rho}+\rho v_{x}=0 \\
\rho \dot{v}+(p-\sigma)_{x}=0 \\
\rho \dot{\varepsilon}+q_{x}+(p-\sigma) v_{x}=0 \\
\frac{\tau_{\sigma}\left[\dot{\sigma}-\frac{8}{15} q_{x}+\frac{7}{3} \sigma v_{x}\right]-\frac{4}{3} \mu v_{x}=-\sigma}{\tau_{q}\left[\dot{q}+\frac{16}{5} q v_{x}-\frac{7}{2}\left(\frac{p}{\rho}\right)_{x} \sigma-\frac{1}{\rho}(p+\sigma) \sigma_{x}+\frac{\sigma}{\rho} p_{x}\right]}+\chi T_{x}=-q
\end{array}\right.
$$

where the dot indicates the material time derivative.

We note that together with the conservations of mass, momentum and energy, we have two new balance laws that govern the behaviour of the extended fields $\sigma$ and $q$. When the underlined terms in (4.3) are negligible the last two equations reduce to the Navier-Stokes and Fourier equations respectively. In particular, this limit can be done using the so-called Maxwellian iteration [10.

4.1. Maxwellian iteration. To reveal the relation between the extended and classical models, a formal iterative scheme known as Maxwellian iteration is applied. The first iterates $q^{(1)}$ and $\sigma^{(1)}$ are calculated from the right-hand sides of the balance laws by putting "zero" ${ }^{t h}$ " iterates: equilibrium values $q^{(0)}=0$ and $\sigma^{(0)}=0$ on the left-hand sides. In the next step the second iterates $q^{(2)}$ and $\sigma^{(2)}$ are obtained from the right-hand sides of the same equations by putting first $q^{(1)}$ and $\sigma^{(1)}$ on their left-hand sides, and so on. Therefore the Maxwellian iteration is substantially an identification of relaxation times and a formal power expansion of the solution in terms of these: a sort of ChapmanEnskog procedure at the macroscopic level. Therefore the Fourier and Navier-Stokes equations are the first approximation of the Maxwellian iteration of the ET balance law system, and therefore they are not true constitutive equations and do not need to satisfy the frame indifference principle. 
We observe that this is true not only for rarefied gas where the Boltzmann equation dictates the special hierarchy (4.2) but also for real gas as was proved recently in [1].

5. The mixture theory. A similar situation occurs in the case of a mixture of fluids for what concerns the Fick's law for the diffusion. Let us consider a mixture of $n$ species and for any constituents $\alpha=1,2, \ldots, n$, we can define the concentrations as:

$$
c_{\alpha}=\frac{\rho_{\alpha}}{\rho}, \quad \rho=\sum_{\alpha=1}^{n} \rho_{\alpha} \quad\left(\sum_{\alpha=1}^{n} c_{\alpha}=1\right) .
$$

There are two possible approaches to the mixture. A classical approach and an extended one.

5.1. Classical theory. In the first case, the $(4+n)$ fields are the $n$ densities of each species $\rho_{\alpha}$, the 3 components of the center of mass velocity $\mathbf{v}$ and one common unique temperature $T$ :

$$
\left(\rho_{\alpha}, \mathbf{v}, T\right), \quad \mathbf{v}=\frac{1}{\rho} \sum_{\alpha=1}^{n} \rho_{\alpha} \mathbf{v}_{\alpha} .
$$

In correspondence we have $(4+n)$ balance laws: the $n$ balance laws of masses of each constituent, the 3 equations of the whole conservation laws of the total momentum and the total energy conservation. The $n$ balance laws of masses can be rewritten as $n-1$ balance equations for the concentrations and the balance law of the total mass of the mixture. Together with the total momentum balance and the total energy balance we have:

$$
\left\{\begin{array}{l}
\dot{\rho}+\rho \operatorname{div} \mathbf{v}=0 \\
\rho \dot{c_{b}}+\operatorname{div} \mathbf{J}_{b}=\tau_{b} \quad(b=1, \ldots, n-1), \\
\rho \dot{\mathbf{v}}-\operatorname{div} \mathbf{t}=0 \\
\rho \dot{\varepsilon}-\mathbf{t} \operatorname{grad} \mathbf{v}+\operatorname{div} \mathbf{q}=0
\end{array}\right.
$$

where

$$
\mathbf{J}_{\alpha}=\rho_{\alpha}\left(\mathbf{v}_{\alpha}-\mathbf{v}\right)
$$

is the diffusion flux vector and $\tau_{b}$ represents the interchange of mass between the constituents in the case of chemical reactions.

The "constitutive equations" associated with the previous balance laws are the NavierStokes's, Fourier's and Fick's laws (1.4).

5.2. The extended theory of mixture. A description of simple homogeneous mixtures in the context of rational thermodynamics relies on the postulate that each constituent obeys the same balance laws as a single fluid [12. They express rates of change of mass, momentum and energy with appropriate production terms due to mutual interaction between the constituents:

$$
\begin{aligned}
& \frac{\partial \rho_{\alpha}}{\partial t}+\operatorname{div}\left(\rho_{\alpha} \mathbf{v}_{\alpha}\right)=\tau_{\alpha}, \\
& \frac{\partial\left(\rho_{\alpha} \mathbf{v}_{\alpha}\right)}{\partial t}+\operatorname{div}\left(\rho_{\alpha} \mathbf{v}_{\alpha} \otimes \mathbf{v}_{\alpha}-\mathbf{t}_{\alpha}\right)=\mathbf{m}_{\alpha} \quad(\alpha=1,2, \ldots, n), \\
& \frac{\partial\left(\frac{1}{2} \rho_{\alpha} v_{\alpha}^{2}+\rho_{\alpha} \varepsilon_{\alpha}\right)}{\partial t}+\operatorname{div}\left\{\left(\frac{1}{2} \rho_{\alpha} v_{\alpha}^{2}+\rho_{\alpha} \varepsilon_{\alpha}\right) \mathbf{v}_{\alpha}-\mathbf{t}_{\alpha} \mathbf{v}_{\alpha}+\mathbf{q}_{\alpha}\right\}=e_{\alpha},
\end{aligned}
$$


where $\varepsilon_{\alpha}, \mathbf{t}_{\alpha}$ and $\mathbf{q}_{\alpha}$ are respectively the internal energy, the stress tensor and the heat flux of the $\alpha$-constituent of the mixture.

Due to the conservations of mass, momentum and energy of the total mixture, the production terms must satisfy the following relations:

$$
\sum_{\alpha=1}^{n} \tau_{\alpha}=0 ; \quad \sum_{\alpha=1}^{n} \mathbf{m}_{\alpha}=\mathbf{0} ; \quad \sum_{\alpha=1}^{n} e_{\alpha}=0 .
$$

One may obtain from (5.2) conservation laws of mass, momentum and energy of the mixture (as a whole) through the summation of appropriate balance laws and introduction of the following quantities:

$$
\begin{array}{ll}
\rho=\sum_{\alpha=1}^{n} \rho_{\alpha} & \text { total mass density, } \\
\mathbf{v}=\frac{1}{\rho} \sum_{\alpha=1}^{n} \rho_{\alpha} \mathbf{v}_{\alpha} & \text { mixture velocity, } \\
\mathbf{u}_{\alpha}=\mathbf{v}_{\alpha}-\mathbf{v} \quad\left(\sum_{\alpha=1}^{n} \rho_{\alpha} \mathbf{u}_{\alpha}=\mathbf{0}\right) & \text { diffusion velocity, } \\
\mathbf{t}=\sum_{\alpha=1}^{n}\left(\mathbf{t}_{\alpha}-\rho_{\alpha} \mathbf{u}_{\alpha} \otimes \mathbf{u}_{\alpha}\right) & \text { stress tensor, } \\
\varepsilon=\frac{1}{\rho} \sum_{\alpha=1}^{n} \rho_{\alpha}\left(\varepsilon_{\alpha}+\frac{1}{2} u_{\alpha}^{2}\right) & \text { internal energy, } \\
\mathbf{q}=\sum_{\alpha=1}^{n}\left\{\mathbf{q}_{\alpha}+\rho_{\alpha}\left(\varepsilon_{\alpha}+\frac{1}{2} u_{\alpha}^{2}\right) \mathbf{u}_{\alpha}-\mathbf{t}_{\alpha} \mathbf{u}_{\alpha}\right\} & \text { flux of internal energy. }
\end{array}
$$

In such a way the conservation laws for the whole mixture read in the same form of a single fluid (1.3):

$$
\begin{aligned}
& \frac{\partial \rho}{\partial t}+\operatorname{div}(\rho \mathbf{v})=0 \\
& \frac{\partial(\rho \mathbf{v})}{\partial t}+\operatorname{div}(\rho \mathbf{v} \otimes \mathbf{v}-\mathbf{t})=\mathbf{0} \\
& \frac{\partial\left(\frac{1}{2} \rho v^{2}+\rho \varepsilon\right)}{\partial t}+\operatorname{div}\left\{\left(\frac{1}{2} \rho v^{2}+\rho \varepsilon\right) \mathbf{v}-\mathbf{t} \mathbf{v}+\mathbf{q}\right\}=0 .
\end{aligned}
$$

Due to the constraints (5.3) it is convenient to rewrite the system (5.2) as the block of conservation laws (5.4) and the balance laws for the first $n-1$ constituents $(b=$ $1,2, \ldots, n-1)$ :

$$
\begin{aligned}
& \frac{\partial \rho_{b}}{\partial t}+\operatorname{div}\left(\rho_{b} \mathbf{v}_{b}\right)=\tau_{b} ; \\
& \frac{\partial\left(\rho_{b} \mathbf{v}_{b}\right)}{\partial t}+\operatorname{div}\left(\rho_{b} \mathbf{v}_{b} \otimes \mathbf{v}_{b}-\mathbf{t}_{b}\right)=\mathbf{m}_{b} \quad(b=1, \ldots, n-1) ; \\
& \frac{\partial\left(\frac{1}{2} \rho_{b} v_{b}^{2}+\rho_{b} \varepsilon_{b}\right)}{\partial t}+\operatorname{div}\left\{\left(\frac{1}{2} \rho_{b} v_{b}^{2}+\rho_{b} \varepsilon_{b}\right) \mathbf{v}_{b}-\mathbf{t}_{b} \mathbf{v}_{b}+\mathbf{q}_{b}\right\}=e_{b} .
\end{aligned}
$$

The unknown fields of the system (5.4) and (5.5) are

$\mathbf{u} \equiv\left(\rho_{\alpha}, \mathbf{v}_{\alpha}, T_{\alpha}\right)^{T}(\alpha=1,2, \ldots, n), \quad$ or $\quad \mathbf{u} \equiv\left(\rho, c_{b}, \mathbf{v}, \mathbf{v}_{b}, T, T_{b}\right)^{T} \quad(b=1,2, \ldots, n-1)$, where $T$ is an appropriate average temperature [16], [15]. We observe that in the classical case (5.1) we omitted the last two blocks of equations (5.5) 2,3 and that the field is simply 
given by

$$
\mathbf{u} \equiv\left(\rho, c_{b}, \mathbf{v}, T\right)^{T} \quad(b=1,2, \ldots, n-1) .
$$

The first constraint for the production terms comes from the requirement of Galilean invariance of the balance equations [13], and we have the complete velocity dependence [14, [15]:

$$
\begin{aligned}
\tau_{b} & =\hat{\tau}_{b}, \\
m_{b}^{j} & =\hat{\tau}_{b} v^{j}+\hat{m}_{b}^{j}, \\
e_{b} & =\hat{\tau}_{b} \frac{v^{2}}{2}+\hat{m}_{b}^{k} v^{k}+\hat{e}_{b},
\end{aligned}
$$

where the hat quantities are velocity-independent.

Another important restriction comes from the entropy inequality, which in the case without chemical reaction implies [14, 15]:

$$
\hat{\mathbf{m}}_{b}=-\sum_{c=1}^{n-1} \psi_{b c}\left(\frac{\mathbf{u}_{c}}{T_{c}}-\frac{\mathbf{u}_{n}}{T_{n}}\right), \quad \hat{e}_{b}=-\sum_{c=1}^{n-1} \theta_{b c}\left(-\frac{1}{T_{c}}+\frac{1}{T_{n}}\right),
$$

where $\psi_{b c}$ and $\theta_{b c}$ are positive semi-definite matrix functions of $\rho_{\alpha}$ and $T_{\alpha}$.

5.3. Maxwellian iteration for the mixture. We have see that the momentum and energy balance for the constituents $(5.5)_{2,3}$ are not present in the classical approach. The question is which is the classical counterpart of (5.5) 2,3 ?

It is extremely interesting to observe that applying the Maxwellian iteration scheme to the momentum equation of single constituents $(5.5)_{2}$ after some simple manipulations, we obtain Fick's law (1.4) 3 [7], [15].

While we also have as a limit of energy balances of each constituent (15.5) 3 a new nonlocal "constitutive equation" for the temperature differences similar to Fick's laws that is not present in the literature [15], 16]:

$$
\Theta_{a}=T_{a}-T=-\sum_{b=1}^{n-1} M_{a b} r_{b} \operatorname{div} \mathbf{v}
$$

where $M_{a b}$ is a positive definite phenomenological matrix and $r_{b}$ is given for perfect gases by

$$
r_{b}=\rho_{b} c_{V}^{(b)}\left(\gamma_{b}-\gamma_{n}\right)
$$

The $\gamma_{b}$ and $c_{V}^{(b)}$ are respectively the ratio of specific heats and the specific heat at constant volume of each constituent. Equation (5.6) is not obtained in classical theory, and it is interesting to observe that maybe this is due to the fact that if the gas has constituents with the same degree of freedom, i.e. the same $\gamma$, then $r_{b} \equiv 0$ and all the temperatures are equal in the first Maxwellian iteration. In this last case, in order to reveal differences of the temperature between constituents it is necessary for us to go further to the second order in the Maxwellian iteration. This was done in a recent paper by Ruggeri and Simić [17. 
5.4. Darcy' law in porous media. Another simple example in the context of mixture theory is that famous Darcy's equation for porous media in which the relative velocity between the fluid part $\mathbf{v}_{F}$ and the solid part $\mathbf{v}_{S}$ is proportional to the pressure gradient in the fluid (see, e.g., [18]):

$$
\operatorname{grad} p_{F}=-\frac{k}{\mu}\left(\mathbf{v}_{S}-\mathbf{v}_{F}\right),
$$

where $k$ and $\mu$ are respectively the permeability and the viscosity.

It is well known that Darcy's law is in reality an approximation to the balance of linear momentum for the fluid that is flowing through the porous solid which is treated as a rigid body; i.e., (5.7) is a limit case of (see [19]):

$$
\rho_{F} \dot{\mathbf{v}}_{F}+\operatorname{grad} p_{F}=-\frac{k}{\mu}\left(\mathbf{v}_{S}-\mathbf{v}_{F}\right) .
$$

6. Maxwellian iteration and entropy principle. We have seen that, using the Maxwellian iteration, we can obtain by extended thermodynamics (at least formally) the usual non-local constitutive equations of classical theory. Therefore the parabolic systems of classical theories appear, from the physical point of view, as approximations of hyperbolic systems when some relaxation times are negligible:

- Fourier's and Navier-Stokes's laws as a limit case of moments equations of extended thermodynamics;

- Fick's law as a limit case of momentum equations of each species in a mixture with single temperature;

- The new diffusion equation for difference of temperatures in mixture with multitemperature as a limit case of energy balance of each constituent.

- Darcy's law for porous material is a limit case of the momentum equation.

Although the previous non-local equations are not constitutive equations but approximations of balance laws the non-local equations are very useful. In many applications the relaxation times are negligible and they are relevant only in limiting situations: rarefied gas, low temperature, high frequencies, etc. Another advantage of the non-local approximation is that in this limit we are able to measure non-observable quantities such as heat flux, viscosity stress and in particular the velocity and the temperature of each species in a mixture of fluids.

Clearly a major open problem in this framework is the rigorous proof of the convergence of the solutions via Maxwellian iterations.

To make a little step toward this important result we have first of all to focus our attention to another very subtle point: is the entropy principle preserved in the Maxwellian iteration scheme? In other words: if the "full" hyperbolic theory satisfies the entropy inequality are we sure that the corresponding parabolic limit satisfies automatically a suitable entropy inequality? From the mathematical point of view it is possible to prove a simple linear counterexample that Costas Dafermos presented to me [20]: Let $v \in R^{m}, \quad w \in R^{n}$

$$
\begin{aligned}
& \partial_{t} v_{i}+\lambda A_{i \alpha} \partial_{t} w_{\alpha}+\mu \partial_{x} v_{i}+B_{i \alpha} \partial_{x} w_{\alpha}=0 \\
& \partial_{t} w_{\alpha}+\lambda A_{\alpha i} \partial_{t} v_{i}+B_{\alpha i} \partial_{x} v_{i}=-w_{\alpha}
\end{aligned}
$$


$\left(\lambda, \mu, A_{i \alpha}, B_{i \alpha}\right.$ constant and $\left.i=1,2, \ldots, m ; \alpha=1,2, \ldots, n\right)$. Putting $\boldsymbol{w}=\mathbf{0}$ in the left side,

$$
\begin{aligned}
& \partial_{t} v_{i}+\mu \partial_{x} v_{i}=0, \\
& \lambda A_{\alpha i} \partial_{t} v_{i}+B_{\alpha i} \partial_{x} v_{i}=-w_{\alpha}
\end{aligned}
$$

eliminating $\partial_{t} v_{i}$

$$
w_{\alpha}=\left(\lambda \mu A_{\alpha i}-B_{\alpha i}\right) \partial_{x} v_{i} .
$$

Substituting into the first equation (6.1):

$$
\partial_{t} v_{j} \simeq-B_{j \alpha} \partial_{x} w_{\alpha}=\left(B_{\alpha i} B_{j \alpha}-\lambda \mu A_{\alpha i} B_{j \alpha}\right) \partial_{x x} v_{i} .
$$

Fix $\boldsymbol{A}$ and $\boldsymbol{B}$, take $|\lambda| \ll 1$, so entropy $\simeq\left(|\mathbf{v}|^{2}+\left|\mathbf{w}^{2}\right|\right) / 2$ is convex, then choose $|\mu| \gg 1$ with a sign that destroys positive definiteness of the parabolic limit.

We need therefore to add other physical requirements. We want to prove that a sufficient condition is to add to the convexity of entropy also the requirement that the process is not far from equilibrium. As is well known, for a system of balance laws (1.2) endowed with a supplementary entropy law:

$$
\partial_{t} h^{0}+\partial_{i} h^{i}=\Sigma \leq 0
$$

with a convex entropy density $h^{0}$ with respect to the densities field $\mathbf{u} \equiv \mathbf{F}^{0}$, we can be put it in a symmetric form by choosing the main field $\mathbf{u}^{\prime}=\partial h^{0} / \partial \mathbf{u}$. In fact, Boillat in the context of the classical approach 21 and Ruggeri and Strumia in a covariant formulation 22] proved that there exist four potentials

$$
h^{\prime \alpha}=\mathbf{u}^{\prime} \cdot \mathbf{F}^{\alpha}-h^{\alpha}, \quad \alpha=0,1,2,3
$$

such that

$$
\mathbf{F}^{\alpha}=\frac{\partial h^{\prime \alpha}}{\partial \mathbf{u}^{\prime}}, \quad \Sigma=\mathbf{u}^{\prime} \cdot \mathbf{f} .
$$

Inserting this into (1.2), the original system becomes symmetric with Hessian matrices (note that $h^{\prime 0}$ is the Legendre transform of $h^{0}$ and therefore a convex function of the dual field $\mathbf{u}^{\prime}$ ):

$$
h_{\mathbf{u}^{\prime} \mathbf{u}^{\prime}}^{\prime \alpha} \partial_{\alpha} \mathbf{u}^{\prime}=\mathbf{f} \quad\left(x^{0}=t, \quad \partial_{\alpha}=\partial / \partial x^{\alpha}\right),
$$

where

$$
h_{\mathbf{u}^{\prime} \mathbf{u}^{\prime}}^{\prime \alpha}=\frac{\partial^{2} h^{\prime \alpha}}{\partial \mathbf{u}^{\prime} \partial \mathbf{u}^{\prime}}
$$

If we split the system into two blocks of $m$ conservation equations and of $n$ balance laws, and in correspondence we represent $\mathbf{u}^{\prime} \equiv(\mathbf{v}, \mathbf{w})$, it was proved in [23] that the equilibrium manifold in the main field components is the hyperplane $\mathbf{w}=0$. In extended thermodynamics, the most interesting case is when the processes are not far from equilibrium. In this case, if we consider one space dimension we can write without loss of generality that

$$
h^{\prime}=h^{\prime 0}=\frac{1}{2}|\mathbf{w}|^{2}+h_{e q}^{\prime}(\mathbf{v}),
$$

and therefore the symmetric system (6.3) assumes the form:

$$
\begin{aligned}
& \mathbf{H} \partial_{t} \mathbf{v}+\mathbf{A} \partial_{x} \mathbf{v}+\mathbf{B} \partial_{x} \mathbf{w}=0, \\
& \partial_{t} \mathbf{w}+\mathbf{B}^{T} \partial_{x} \mathbf{v}+\mathbf{C} \partial_{x} \mathbf{w}=-\mathbf{L} \mathbf{w},
\end{aligned}
$$


where $\mathbf{v} \in R^{m}, \mathbf{w} \in R^{n}, \mathbf{H} \equiv h_{\mathbf{v} \mathbf{v}}^{\prime}(m \times m) \in \operatorname{Sym}^{+}$(symmetric positive definite matrix), $\mathbf{A} \equiv k_{\mathbf{v v}}(m \times m) \in \operatorname{Sym}$ (symmetric matrices), $\mathbf{B} \equiv k_{\mathbf{v w}}(m \times n), \mathbf{C} \equiv k_{\mathbf{w w}}(n \times n) \in$ Sym and $k=h^{\prime 1}(\mathbf{v}, \mathbf{w})$. Moreover $\mathbf{L} \equiv \mathbf{L}(\mathbf{v})(n \times n) \in$ Sym $^{+}$because of the residual inequality $\Sigma=-\mathbf{w}^{T} \mathbf{L w} \leq 0$.

Putting $\mathbf{w}=0$ into (6.6) we have

$$
\overline{\mathbf{B}}^{T} \partial_{x} \mathbf{v}=-\mathbf{L} \mathbf{w}
$$

where $\overline{\mathbf{B}} \equiv \mathbf{B}(\mathbf{v}, \mathbf{0})$. Then substituting into (6.5) we obtain as the first Maxwellian iteration:

$$
\mathbf{H} \partial_{t} \mathbf{v} \simeq \mathbf{D} \partial_{x x} \mathbf{v}
$$

where the diffusion matrix is given by

$$
\mathbf{D}=\overline{\mathbf{B}} \mathbf{L}^{-1} \overline{\mathbf{B}}^{T} \in S y m^{+} .
$$

Consequently the entropy principle is preserved in the passage from the hyperbolic system to the parabolic limit.

In the general case, as we have observed by the counterexample, the entropy principle is not necessarily preserved. In this case instead of (6.5), (6.6) we have:

$$
\begin{aligned}
& \mathbf{H} \partial_{t} \mathbf{v}+\mathbf{G} \partial_{t} \mathbf{w}+\mathbf{A} \partial_{x} \mathbf{v}+\mathbf{B} \partial_{x} \mathbf{w}=0, \\
& \mathbf{G}^{T} \partial_{t} \mathbf{v}+\mathbf{K} \partial_{t} \mathbf{w}+\mathbf{B}^{T} \partial_{x} \mathbf{v}+\mathbf{C} \partial_{x} \mathbf{w}=-\mathbf{L} \mathbf{w}
\end{aligned}
$$

with $\mathbf{G} \equiv h_{\mathbf{v w}}^{\prime}(m \times n)$ and $\mathbf{K} \equiv h_{\mathbf{w w}}^{\prime}(n \times n) \in S y m^{+}$. After Maxwellian iteration we obtain a parabolic system similar to (6.7) but with a diffusion matrix $\mathbf{D}$ :

$$
\mathbf{D}=\overline{\mathbf{B}} \mathbf{L}^{-1} \overline{\mathbf{B}}^{T}-\overline{\mathbf{B}} \mathbf{L}^{-1} \overline{\mathbf{G}}^{T} \overline{\mathbf{H}}^{-1} \overline{\mathbf{A}}
$$

that does not have in general a definite sign. The most general system therefore for which the entropy principle is preserved in the parabolic limit is the one for which $\mathbf{D}$ given by (6.11) belongs to $\mathrm{Sym}^{+}$. We call this special system preserving entropy systems.

A simple preserving entropy system is the one for which $h^{\prime}$ is given by the sum of two functions, those of only $\mathbf{v}$ and one of $\mathbf{w}$. The interesting case of the process not far from equilibrium (6.4) is a special class of this class. In fact in this case we have $\mathbf{G} \equiv 0$ and the diffusion matrix (6.11) reduces to (6.8) and becomes symmetric positive definite.

7. The mathematical situation. From the mathematical point of view it seems that on the contrary that hyperbolic systems are particular cases of parabolic ones. The prototype is Burgers equation with artificial viscosity to restore the uniqueness of weak solutions:

$$
u_{t}+u u_{x}=\underline{\mu u_{x x}} .
$$

Moreover, roughly speaking, parabolic equations seem to be more realistic because usually there exist regular solutions, to the contrary of hyperbolic ones, for which regular solutions soon become shocks or blow up. I want now to show that this is not completely true also from the mathematical point of view. The first question is in reality a misunderstanding due to the confusion between systems and subsystems. I want to explain this 


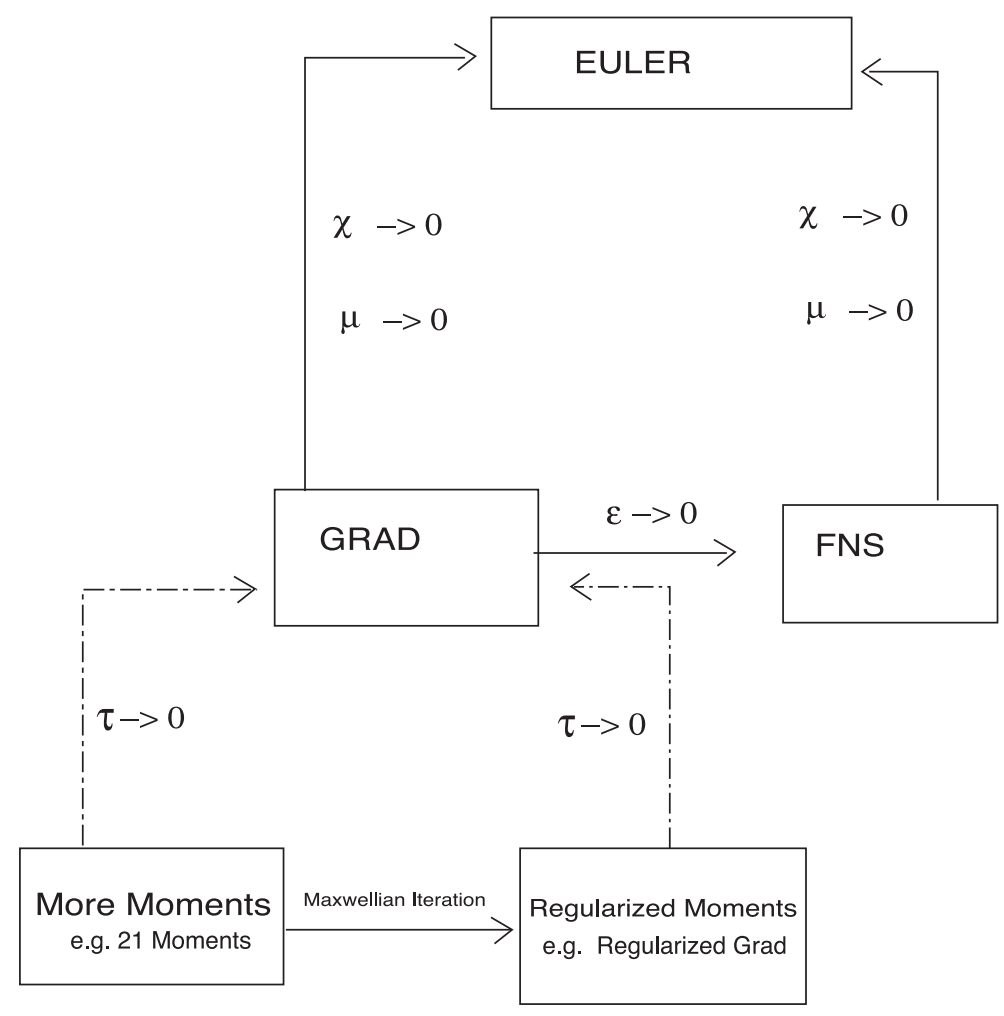

FIG. 1. Systems and subsystems and the parabolic limit.

with the simple example of the Grad system (4.3). We have to recall that the relaxation times $\tau_{\sigma}, \tau_{q}$ are proportional to the viscosity and heat conductivity respectively [7]:

$$
\tau_{\sigma} \propto \varepsilon \mu, \quad \tau_{q} \propto \varepsilon \chi
$$

where $\varepsilon$ is related to the Knudsen number. If we look at Figure 1 and (4.3) we can see that effectively the Euler fluid subsystem is a particular case of the Navier and Fourier systems when $\mu$ and $\chi$ vanish. Nevertheless also Euler is a subsystem of the Grad system and moreover the Navier-Stokes-Fourier system is a particular case of the Grad system when $\varepsilon$ tends to zero. Therefore the confusing point in the literature is that the Euler system is a particular subsystem of both the Navier-Stokes-Fourier system and the Grad system. This situation is also valid if we take ET with many moments. When we apply the Maxwellian iteration we have as a parabolic counterpart the so-called regularized moments [24]. In any case, given an $n$-moment system, all the previous moments are principal subsystems according to the general mathematical nesting structure which was found by Boillat and Ruggeri [23. Moreover all the parabolic limits also can be considered as approximations of the hyperbolic system as sketched in Figure 1.

7.1. Qualitative analysis. On the other hand the question that parabolic systems can have smooth solutions in contrast to hyperbolic systems is also not true because all 
the ET examples are hyperbolic systems with dissipation. It is well known that systems of balance laws endowed with a convex entropy law, and dissipative, the so-called Kawashima-Shizuta K-condition [25] becomes a sufficient condition for the existence of global smooth solutions provided the initial data are sufficiently smooth (Hanouzet and Natalini [26], Wen-An Yong [27], Bianchini, Hanouzet and Natalini [28]; see also the Dafermos book [29]):

Theorem 7.1 (Global existence). Assume that the system of balance laws is strictly dissipative and the $\mathrm{K}$-condition is satisfied. Then there exists $\delta>0$, such that, if $\|\mathbf{u}(x, 0)\|_{2} \leq \delta$, there is a unique global smooth solution, which satisfies

$$
\mathbf{u} \in \mathcal{C}^{0}\left([0, \infty) ; H^{2}(\mathbb{R}) \cap \mathcal{C}^{1}\left([0, \infty) ; H^{1}(\mathbb{R})\right) .\right.
$$

Moreover Ruggeri and Serre have proved in the one-dimensional case that the constant states are stable [30]:

TheOREM 7.2 (Stability of constant state). Under the natural hypothesis of strongly convex entropy, strict dissipativeness, genuine coupling and "zero mass" initial for the perturbation of the equilibrium variables, the constant solution stabilizes

$$
\|\mathbf{u}(t)\|_{2}=O\left(t^{-1 / 2}\right) .
$$

Recently Lou and Ruggeri [31] have observed that the weaker K-condition in which we require the K-condition only for the right eigenvectors corresponding to genuine nonlinear systems is a necessary (but not sufficient) condition for the global existence of smooth solutions. This is the case of ET in classical [32] and in relativity [33], 34] and in the case of a mixture with multi-temperatures [14].

8. Conclusions. In conclusion, extended thermodynamics seems to indicate in a clear manner that non-local relations are not constitutive equations but approximations of balance laws. The true constitutive equations are in local form and they obey the material frame difference. The physical systems are hyperbolic in agreement with the relativity principle that any disturbance propagate with finite speed. Nevertheless the usual Fourier, Navier-Stokes, Fick, Darcy and other non-local equations are useful to measure non-observable quantities and they are good approximations in many practical problems. The Maxwellian iteration preserves the entropy principle at least for processes not far from equilibrium. Hyperbolic systems with dissipation (balance laws with production terms) can have global smooth solutions provided that the initial data are small.

\section{REFERENCES}

[1] C. Truesdell, W. Noll and S. S. Antman, The non-linear field theories of mechanics, Volume 3. Springer, 1-602 (2004). MR0193816 (33:2030)

[2] B. Coleman and W. Noll, The thermomechanics of elastic materials with heat conduction and viscosity, Arch. Rational Mech. Anal. 13, 167-178 (1963). MR0153153 (27:3122)

[3] I. Müller, On the entropy inequality, Arch. Rational Mech. Anal. 26, 118-141 (1967). MR0214336 (35:5187) 
[4] I. Müller, On the frame dependence of stress and heat flux, Arch. Rational Mech. Anal. 45, 241 (1972). MR 1553565

[5] A. Bressan, On relativistic heat conduction in the stationary and nonstationary cases, the objectivity principle and piezoelasticity, Lett. Nuovo Cimento, 33 (4), 108 (1982).

[6] T. Ruggeri, Generators of hyperbolic heat equation in nonlinear thermoelasticity, Rend. Sem. Mat. Padova, 68, 79 (1982). MR702148 (85e:73066)

[7] I. Müller and T. Ruggeri, Rational Extended Thermodynamics, Springer Tracts in Natural Philosophy 37, 2nd Ed., Springer Verlag (1998). MR1632151(99h:80001)

[8] H. Grad, On the kinetic theory of rarefied gases. Comm. Appl. Math. 2, 331 (1949). MR0033674 $(11: 473 \mathrm{a})$

[9] I-S. Liu and I. Müller, Extended thermodynamics of classical and degenerate ideal gases, Arch. Rational Mech. Anal. 83, (4), 285-332 (1983). MR714978 (85j:80001)

[10] E. Ikenberry and C. Truesdell, On the pressures and the flux of energy in a gas according to Maxwell's kinetic theory, J. Rational Mech. Anal. 5, 1 (1956). MR0075725 (17:796c)

[11] T. Arima, S. Taniguchi, T. Ruggeri and M. Sugiyama, Extended thermodynamics of dense gases, Continuum Mech. Thermodyn. DOI 10.1007/s00161-011-0213-x (2011).

[12] C. Truesdell, Rational Thermodynamics, McGraw-Hill, New York, 1969. MR0366236 (51:2484)

[13] T. Ruggeri, Galilean Invariance and Entropy Principle for Systems of Balance Laws. The Structure of the Extended Thermodynamics, Contin. Mech. Thermodyn. 1, 3 (1989). MR1001434 (90c:80003)

[14] T. Ruggeri and S. Simić, On the Hyperbolic System of a Mixture of Eulerian Fluids: A Comparison Between Single and Multi-Temperature Models. Math. Meth. Appl. Sci., 30, 827 (2007). MR2310555 (2008e:35158)

[15] T. Ruggeri and S. Simić, Average temperature and Maxwellian iteration in multitemperature mixtures of fluids. Phys. Rev. E 80, 026317 (2009).

[16] H. Gouin and T. Ruggeri, Identification of an average temperature and a dynamical pressure in a multi-temperature mixture of fluids. Phys. Rev. E 78, 01630, (2008).

[17] T. Ruggeri and S. Simić, Non-equilibrium temperatures in the mixture of gases via Maxwellian iteration. Submitted in Phys. Rev. E. (2012).

[18] K. Wilmanski, Continuum Thermodynamics - Part 1: Foundations. World Scientific, Singapore, 2008. MR2482665 (2010f:74002)

[19] R. Rajakopal, On a Hierarchy of Approximate Models for Flows of Incompressible Fluids through Porous Solids. Mathematical Models and Methods in Applied Sciences Vol. 17, No.2, 215-252 (2007). MR.2292356(2007k:76156)

[20] C. Dafermos, Private Communication (Bologna 2011).

[21] G. Boillat, Sur l'existence et la recherche d'équations de conservation supplémentaires pour les systèmes hyperboliques. C.R. Acad. Sc. Paris, 278-A, 909-912 (1974). Non Linear Fields and Waves. In CIME Course, Recent Mathematical Methods in Nonlinear Wave Propagation, Lecture Notes in Mathematics 1640, T. Ruggeri Ed. Springer-Verlag, 103-152 (1995). MR0342870 (49:7614)

[22] T. Ruggeri and A. Strumia, Main field and convex covariant density for quasi-linear hyperbolic systems. Relativistic fluid dynamics, Ann. Inst. H. Poincaré 34 A, 65 (1981). MR605357 (82b:76077)

[23] G. Boillat and T. Ruggeri, Hyperbolic Principal Subsystems: Entropy Convexity and Subcharacteristic conditions. Arch. Rat. Mech. Anal. 137, 305-320 (1997). MR1463797 (98h:82055)

[24] H. Struchtrup and M. Torrilhon, Regularization of Grad's 13 moment equations: Derivation and linear analysis, Phys. Fluids, 15 pp. 2668-2680, (2003). MR2060065 (2005a:76142)

[25] Y. Shizuta and S. Kawashima, Systems of equations of hyperbolic-parabolic type with applications to the discrete Boltzmann equation, Hokkaido Math. J., 14, 249 (1985). MR798756 (86k:35107)

[26] B. Hanouzet and R. Natalini, Global existence of smooth solutions for partially dissipative hyperbolic systems with a convex entropy. Arch. Rat. Mech. Anal. 16989 (2003). MR2005637(2004h:35135)

[27] Wen-An Yong, Entropy and global existence for hyperbolic balance laws. Arch. Rat. Mech. Anal. 172 , no. 2, 247-266 (2004). MR2058165 (2005c:35195)

[28] S. Bianchini, B. Hanouzet and R.Natalini, Asymptotic Behavior of Smooth Solutions for Partially Dissipative Hyperbolic Systems with a Convex Entropy. Comm. Pure Appl. Math., Vol. 60, 1559 (2007). MR2349349 (2010i:35227)

[29] C. Dafermos, Hyperbolic conservation laws in continuum physics, Springer, Berlin, 2000. MR:1763936(2001m:35212)

[30] T. Ruggeri and D. Serre, Stability of constant equilibrium state for dissipative balance laws system with a convex entropy, Quart. Appl. Math, 62 (1), 163 (2004). MR2032577(2004k:35257) 
[31] J. Lou and T. Ruggeri, Acceleration Waves and Weak Shizuta-Kawashima Condition, Suppl. Rend. Circ. Mat. Palermo Nonlinear Hyperbolic Fields and Waves. A tribute to Guy Boillat, Serie II, Numero 78, pp. 187-200 (2006). MR2210603 (2006k:35254)

[32] T. Ruggeri, Global existence of smooth solutions and stability of the constant state for dissipative hyperbolic systems with applications to extended thermodynamics, in Trends and Applications of Mathematics to Mechanics, STAMM 2002. Springer-Verlag 215, (2005).

[33] T. Ruggeri, Entropy principle and Relativistic Extended Thermodynamics: Global existence of smooth solutions and stability of equilibrium state. Il Nuovo Cimento B, 119 (7-9), 809-821 (2004). Lecture notes of the International Conference in honour of Y. Choquet-Bruhat: Analysis, Manifolds and Geometric Structures in Physics, G. Ferrarese and T. Ruggeri Eds. (2004). MR2136908 (2006g:80002)

[34] T. Ruggeri, Extended Relativistic Thermodynamics. Section inserted in the book of Yvonne Choquet Bruhat, General Relativity and the Einstein equations, pp. 334-340. Oxford Univ. Press, ISBN 9780-19-923072-3, (2009). 